\title{
Computer Analysis of Implanted Cardiac Pacemaker Rhythm
}

\author{
JAA Fairweather $^{1}$, P Johnston ${ }^{2}$, S Luo $^{2}$, PW Macfarlane ${ }^{1}$ \\ ${ }^{1}$ University of Glasgow, Scotland, UK \\ ${ }^{2}$ Cardiac Science Corporation, Deerfield, WI, USA
}

\begin{abstract}
Implanted cardiac pacemakers are becoming more sophisticated. However, accurate detection of pacemaker stimuli is a problem for most ECG machines because of the very short duration of the stimuli, sometimes only $0.3 \mathrm{~ms}$. In addition, the relatively new technique of biventricular pacing involves two closely timed stimuli to activate the ventricles separately. Detection of all stimuli in a paced patient is therefore becoming increasingly difficult for conventional ECG machines.

In order to address this problem, a more recently developed electrocardiograph, namely the Burdick Atria 6100, introduced enhanced front end electronics. This study assessed its accuracy in detecting pacemaker stimuli and assessed the choice of lead for their detection. 51 patients were initially recruited. Five were excluded for various reasons. Pacemaker activity was detected in 45/46 patients and in 16/16 patients with biventricular pacemakers, two closely paced stimuli were detected.
\end{abstract}

\section{Introduction}

More than 239,000 patients in the United Kingdom have pacemakers, with over 30,000 pacemakers implanted annually in this country alone [1]. With an ageing population and widening indications for pacemaker implantation, the use of pacemakers and pacing devices is likely to increase.

The electrocardiogram (ECG) is the most commonly used diagnostic test in clinical medicine [2], and is used in pacemaker follow-up [3] as well as for other reasons $2-5 \%$ of routine diagnostic ECGs are taken from pacemaker patients [4]. Yet pacemakers present special problems in ECG use. Interpretation of paced ECGs is complex and difficult $[5,6]$, especially with the technological complexity of modern pacemakers [7].

Some electrocardiographs produce automated 12-lead ECG reports. This requires the cardiac waveform to be converted from an analogue to digital form, normally at a rate of 500 samples per second (sps), i.e. every $2 \mathrm{~ms}$. However, artificial electronic pacing stimuli can be as brief as $0.2 \mathrm{~ms}$, so many are not detected and displayed on the 12-lead ECG printout. Thus, automated computerised interpretation of ECGs from patients with pacemakers is often inaccurate. Studies examining the performance of automated computer-based ECG reporting demonstrate that pacemakers are responsible for many of the errors made. In one [8], pacemakers were responsible for the second largest number of computer report errors, with a detection sensitivity of only $73.2 \%$. In a separate study [9], pacemakers were the most common cause of error. Three quarters of the 343 ECGs recorded from patients with pacemaker activity were reported inaccurately. This poor performance has resulted in a call for development of better algorithms to cope with paced ECGs [8], with some success reported [4].

An alternative to new algorithm development is an improvement in the sampling rate, which has been achieved by Cardiac Science Corporation in their Burdick Atria 6100 electrocardiograph. This machine can sample at a rate of up to 64,000 sps in two channels.. The availability of this machine has led to the present study which was designed to assess the performance of the new Atria in the detection of pacemaker stimuli. The Atria 6100 was programmed with the University of Glasgow (Uni-G) ECG Analysis Program [2]. Pacing spike detection is undertaken by the ECG machine's inbuilt firmware, while interpretation of the spikes detected is undertaken by the Uni-G program. Currently, the Atria samples at $64,000 \mathrm{sps}$ in Leads II and V2 in order to detect pacing stimuli.

\section{Methods}

\subsection{Patients materials and data collection}

Subjects known to have implanted cardiac pacemakers or pacing devices (e.g. Implantable Cardioverter Defibrillators (ICDs)) were recruited between January and March 2007 from outpatient pacemaker and ICD follow-up clinics and from pacemaker implant theatre lists. Male and female patients of all ages who were wellenough to take part were invited to participate. Informed, written consent was obtained from each subject prior to his or her participation and examination of the relevant 
clinical notes.

Subjects were questioned to gather demographic and personal details. Their clinical notes were consulted to gather specific device details: the type of pacing, classification of their device and the frequency of demand pacing. Where possible, information about the pacing stimuli, such as the actual voltage and duration of pacing pulses, was gathered from the device programmers (manufacturer specific devices used in clinics to interrogate pacemakers and set parameters).

Two standard 12-lead resting ECGs were recorded and printed from each subject (one after the other), following strict technique guidelines [10]. Settings were then altered on the Atria to allow recordings of two channels of high speed data which could be examined and analysed separately in order to measure pacing stimuli amplitudes in leads I, II and V1-V6, which would allow lead comparisons. These high-speed data files were stored on the Atria and subsequently transferred to a personal computer. In view of data transfer limitations, lead II was transferred at 64,000 sps but other pairs of leads, e.g. I, V6 were transferred at 32,000 sps per lead. Subsequently, the ECG data were extracted using a custom made tool. The numerical data points were then presented graphically using Microsoft Excel 2000. Figure 1 is an example of a chart produced.

\subsection{Pacing spike visibility}

To compare the visibility of pacing spikes, ECGs from those subjects with continuous ventricular pacing were analysed. The mean proportion of cardiac cycles with visible spikes was calculated from the two Atria ECGs for all patients and the overall mean proportion found.

\subsection{Accuracy of spike reporting}

Automated reports from the 12-lead ECGs were marked accurate or inaccurate. Reports were judged to be "accurate" if they identified the pacing activity completely correctly, irrespective of other comments, based on the information known about the subject (from clinical notes and details gathered in the pacemaker clinic during routine clinic device interrogation) and the appearance of the 12-lead ECG. If they did not, they were classified as "inaccurate." The report was classified "unknown" where relevant information was not available, or if it could not be established from the ECG findings, e.g. if a single spike were not detected in regular ventricular pacing, and the report therefore said "Demand ventricular pacing", this was judged as "inaccurate".

\subsection{Lead comparisons}

To determine which ECG lead is most able to detect the pacemaker activity, the line graphs of the data points were examined for the presence of pacing spikes (see Figure 1). For each subject, the peak-to-peak amplitude of the first 3 spikes was measured in leads I, II and V1V6, and a mean for each lead calculated. Where more than one spike was present for each cardiac cycle (e.g. in atrio-ventricular sequential pacing), the equivalent stimulus was measured each time to allow meaningful comparison between the leads.

Simple descriptive statistics were examined to identify which lead had the overall largest spike amplitude, and a repeated measures analysis of variance, under the general linear model, was then used to determine if there were significant relationships between the leads, i.e. if one lead generally gave larger amplitudes. The spike measurements of the lead proposed for future use must not have a standard deviation greater than double that of the lead with the lowest pulse amplitude.

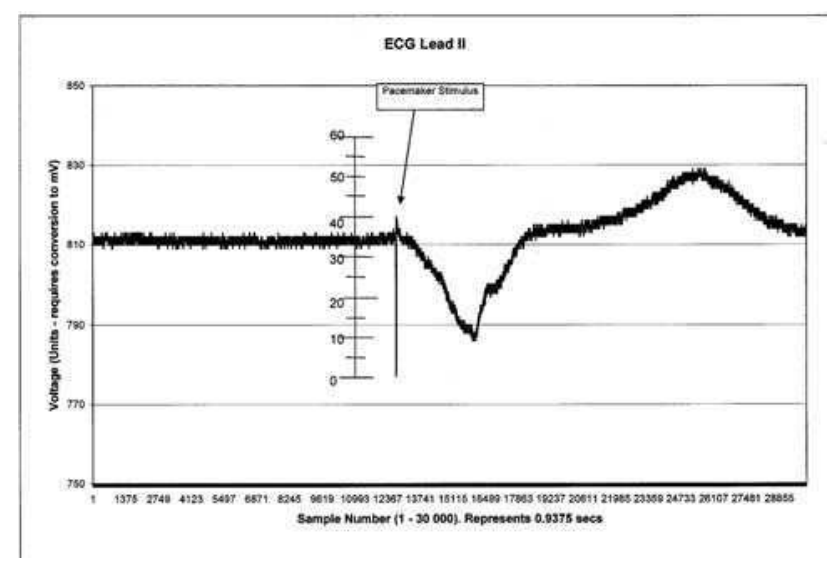

Figure 1. Example of measurement of pacemaker spike amplitude.

\section{Results}

A total of 51 subjects participated. 30 were recruited from pacemaker and ICD clinics, and 21 were recruited as in-patients. One subject was excluded after data collection because his implanted device had no pacing activity. Details of the cohort are given in Table 1 .

\subsection{Pacing spike visibility}

Figure 2 shows an example of the 12-lead resting ECGs recorded on the Atria. 13 subjects had definite continuous ventricular pacing, identified by clinical notes and confirmed by QRS complex examination. In these subjects, the Atria always appropriately displayed a pacing spike before each QRS complex.

Table 1. Characteristics of study patients $(n=50)$ 


\begin{tabular}{|l|l|}
\hline \multicolumn{1}{|l|}{ Age } & \\
\multicolumn{1}{|c|}{ Mean \pm Std Dev } & 63.8 years \pm 14.1 \\
Mean age males & 67.1 years \\
Mean age females & 63.4 years \\
\hline Sex & $35(70 \%)$ \\
Males & $15(50 \%)$ \\
Females & $12(24 \%)$ \\
Pacemaker & $6(12 \%)$ \\
DDD & $3(6 \%)$ \\
DDDR & $4(8 \%)$ \\
VVI & $2(4 \%)$ \\
VVIR & $16(32 \%)$ \\
AAI & $7(14 \%)$ \\
Biventricular pacing & \\
Unknown &
\end{tabular}

\subsection{Accuracy of spike reporting}

4 subjects were excluded from this analysis for technical reasons. The Atria completely correctly reported pacing activity in $82.6 \%(n=38)$ of cases and was inaccurate in $17.4 \%(n=8)$ of cases. Pacemaker activity was detected in $45 / 46$ cases.

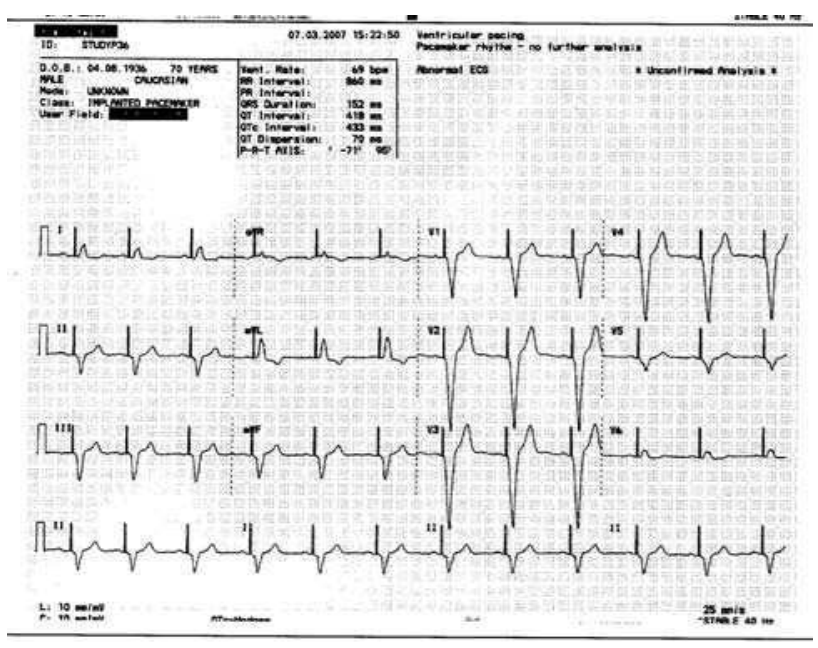

Figure 2. Example of regular ventricular pacing as recorded by the Atria 6100 .

\subsection{Lead position amplitude and variability}

The largest median spike amplitude was found in lead $\mathrm{V} 4$, while the smallest was in V1. The medians for V3, V4, V5, and V6 are all high and relatively similar between $5.49 \mathrm{mV}$ and $6.31 \mathrm{mV}$.

Because of skewed data, statistical analysis first required data transformation (logarithm base 10) to achieve data closer to a Normal Distribution. A repeated measures analysis of variance was then carried out (using Minitab), under the general linear model. Lead was a statistically significant factor in determining amplitude of pacing spikes $(\mathrm{p}<0.0001)$. Tukey comparisons showed that there was no significant difference between leads I, V3, V4, V5, and V6 ( $P$ values all $>0.05)$.

The variability of each lead (for all subjects), shown by the standard deviation of spike amplitudes, was calculated and although the variability of some leads is greater than others, the largest standard deviation (Lead I, $\mathrm{SD}=21.44 \mathrm{mV}$ ) is less than double that of the smallest $($ Lead V1, SD $=12.92 \mathrm{mV})$ - hence, from a statistical standpoint, equal variability can be accepted.

\subsection{Biventricular pacing}

16 subjects had biventricular pacing. On the standard 12-lead ECG printouts, single ventricular spikes were visible. The Atria reported "ventricular pacing" in all cases, which, although accurate, is not an adequate description. However, examination of the 32,000 sps charts shows that, in every case, two ventricular spikes were visible (as in Figure 3), with $2.28 \mathrm{~ms}$ to $10.95 \mathrm{~ms}$ delay between the ventricular stimuli.

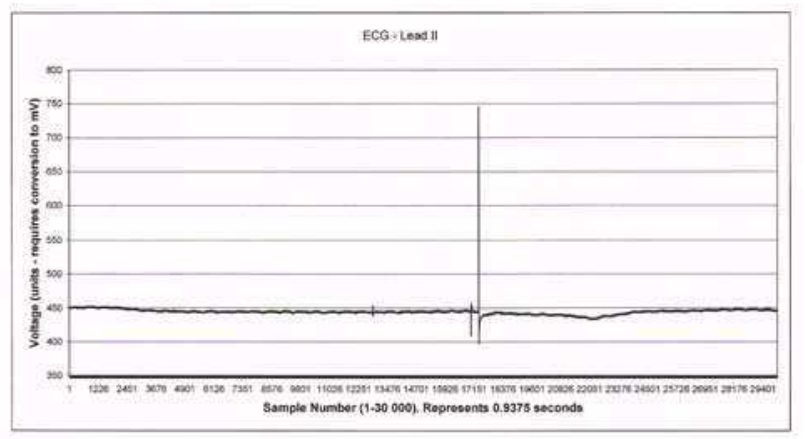

Figure 3. An example of biventricular pacing. Three stimuli, one atrial and two ventricular, can be seen.

\section{Discussion and conclusions}

The results add to evidence on the performance of automated computer based ECG rhythm analysis related specifically to pacemakers. One recent study found that only $24.8 \%$ of ECGs with pacemaker activity were reported accurately, using GE Healthcare Technology 12 lead electrocardiographs with a standard software algorithm for rhythm diagnosis (005C, version 19) [9]. Another study, using the 12SL computer software for analysis, found that $78.5 \%$ of paced ECGs were recognised, but many were still not reported fully. [8].

In this study, pacemaker reporting sensitivity in terms 
of diagnostic accuracy was found to be $82.6 \%$. A higher sensitivity may be achieved in future by using a more advanced spike detection algorithm applied to two (or perhaps more) leads with a higher amplitude pacemaker stimulus than that of lead II.

\subsection{Lead position amplitude and variability}

Selection of the lead which gives the best chance of detecting pacers was then made. Tukey's comparisons show no statistically significant difference between leads I, V3, V4, V5, and V6. In view of this, along with the lack of any significant difference between leads I, V3, $\mathrm{V} 4$, V5 and V6, and the fact that V4 gives the greatest median value, it is recommended that lead V4 be chosen for detecting pacemaker stimuli, if only one lead is used.

\subsection{Biventricular pacing}

Biventricular pacing gives rise to new challenges in the assessment of pacing function and requires a greater understanding of its effect on the ECG [10]. As far as is known, this is the first demonstration (Figure 3 ) of the detection of distinct biventricular pacing spikes using a standard, albeit enhanced, ECG machine.

Although two spikes might not always be visible in biventricular pacing, the fact that two distinct spikes are seen in all 16 cases means that there is potential for developing an algorithm possibly involving analysis of QRS morphology, which would allow automated detection of biventricular pacing, Furthermore, in light of the advocated resurgence of 12 lead ECG use in CRT follow-up $[3,10,11]$, technology which is able to report biventricular pacing will prove to be of great clinical use.

\subsection{Study limitations}

A larger cohort allowing large subgroup analyses may have revealed potentially clinically relevant information, e.g. the effect of age, sex, or body mass index on the choice of most suitable lead was not assessed.

Automated ECG reports are clinically useful [8], and can speed up ECG interpretation and reduce errors [12]. Altering the programming of the Atria based on the results of this study, so that high-speed pacing detection is undertaken by Lead V4 rather than the historical Lead II may yield improved sensitivity and thus clinical usefulness of automated reports in the future. The development of logic to report biventricular pacing which may now be possible because distinct ventricular spikes have been found - may further improve the reporting accuracy for pacing devices.

\section{References}

[1] Cunningham AD. Report for the European Heart Rhythm Association Central Cardiac Audit Database. Central Cardiac Audit Database. 2004. http://www.ccad.org.uk

[2] Macfarlane PW, Devine B, Clark E. The University of Glasgow (Uni-G) ECG Analysis Program. Computers in Cardiol. 2005; 32:451-454.

[3] Barold SS, Levine PA, Eli Ovsy Shcher I. The Paced 12Lead Electrocardiogram Should No Longer Be Neglected in Pacemaker Follow-up. J. Pacing Clin. Electrophysiol. 2001;24:1455-58.

[4] Helfenbein ED, Lindauer JM, Zhou SH, Gregg RE, Herleikson EC. A Software-based Pacemaker Pulse Detection and Paced Rhythm Classification Algorithm. J Electrocardiol. 2002; 35 (Supplement): 95-103.

[5] Kuo Y, Guo Y, Jin J, Huang Y, Luo Z. Interpretation of Paced ECD; Proceedings of the $20^{\text {th }}$ Annual Conference of the IEEE Engineering in medicine and Biology Society. 1998; 20:210-3.

[6] Rosner MH, Brady WJ. The Electrocardiographic Diagnosis of Acute Myocardial Infarction in patients with ventricular paced rhythms; Am J Emerg Med. 1999; 17:182-5.

[7] Stone KR, McPherson CA. Assessment and management of patients with pacemakers and implantable cardioverter defibrillators. Crit. Care Med. 2004;32 (Supplement) S155S165.

[8] Guglin ME, Thatai D. Common Errors in computer electrocardiogram interpretation. Internat. $\mathrm{J}$ Cardiol. 2006;106:232-7.

[9] Poon K, Okin PM, Kligfield P. Diagnostic performance of a computer-based ECG rhythm algorithm. J Electrocardiol. 2005;38:235-8.

[10] Steinberg JS, Maniar P.B, Higgins SL. et al. Noninvasive Assessment of the Biventricular Pacing System. Ann Noninvasive Electrocardiol. 2004;9: 58-70.

[11] Barold SS, Herweg B, Giudici M. Electrocardiographic follow up of biventricular pacemakers. Ann Nonimvasive Electrocardiol. 2005;10:231-55.

[12] Brailer DJ, Kroch E, Pauly MV. The Impact of computerassisted test interpretation on physician decision making: the case of electrocardiograms. Med. Dec. Making. 1997; 17: 80-6.

Address for correspondence:

Professor Peter W. Macfarlane,

Electrocardiology Group,

Faculty of Medicine - University of Glasgow

Level 4, QEB,

Royal Infirmary,

10 Alexandra Parade,

Glasgow G31 2ER

Scotland, UK

peter.w.macfarlane@clinmed.gla.ac.uk 